\title{
Nonperturbative stopping-power calculation for bare and neutral hydrogen incident on He
}

\author{
P. L. Grande* and G. Schiwietz \\ Hahn-Meitner-Institut Berlin GmbH, Department P3, Glienicker Strasse 100, D-1000 Berlin 39, Federal Republic of Germany
}

(Received 21 September 1992)

\begin{abstract}
The electronic stopping power of hydrogen beams penetrating $\mathrm{He}$ gas has been calculated by solving the time-dependent Schrödinger equation. Special attention has been given to low incident energies where capture and loss of the projectile electron are the most important energy-loss mechanisms. These mechanisms are included in a consistent theoretical treatment and good agreement with experimental data has been obtained for high- as well as for low-energy projectiles in a gas target.
\end{abstract}

PACS number(s): $34.50 . \mathrm{Bw}, 79.20 . \mathrm{Nc}, 61.80 . \mathrm{Mk}, 34.10 .+\mathrm{x}$

\section{INTRODUCTION}

At low energies $(E<25 \mathrm{keV} / \mathrm{amu})$, direct measurements of the electronic stopping power are rather scarce [1] since the energy losses involved in such cases are very small. Currently there is no $a b$ initio theory that accurately describes the low-energy stopping processes for ions in gases or insulators. For this reason, most of the stopping-power tabulations [2-4] and semiempirical models [5] extrapolate their results from intermediate and high energies to lower ones, using velocity dependencies that are based on extremely simple models [6,7]. In the low-velocity regime, the energy loss is basically dominated by electron capture and loss of projectile electrons and, in the case of metals, is due to the excitation of a small portion of electrons near the Fermi level to empty states in the conduction band. According to the Firsov model [6] and the Lindhard electron-gas theory [7], both mechanisms yield a linear dependence of the electronic stopping power with the projectile velocity. For metals, this behavior is well corroborated by other calculations [8-10] and is a consequence of the minimum energy transfer being zero in these cases. On the other hand, in the case of gases or insulators, the electronic stopping power should fall off faster at low ion velocities, since, in most cases, there is a nonzero minimum energy transfer.

Previous theoretical calculations were restricted to low-energy ions penetrating conducting solids [8-10] or to intermediate and high projectile velocities on gases [11]. In the following, we provide an improved stopping-power calculation that accounts for the basic stopping processes in gases, namely electron capture and loss of projectile electrons, as well as target ionization and excitation for different charge states of the incoming ion. This will be done by solving numerically the timedependent Schrödinger equation for the electronic system through the coupled-channel method $[11,12]$. Only recently a stopping-power calculation of this type was performed for the $\mathbf{H}^{+}$charge-state fraction at intermediate and high energies [11]. In the present work, we extend our previous calculations by including one projectilecentered state, in order to improve the treatment of the capture process that is fundamental for the electronic stopping power at low energies. This enables us to calcu- late the electronic stopping power of protons on $\mathrm{He}$ atoms with high accuracy. Unless indicated otherwise, we use atomic units throughout this work.

\section{THEORY}

The principle of the present calculation is based on the impact-parameter method $[13,14]$. The projectile (bare or neutral) following a classical trajectory provides a timedependent perturbation on the target electrons. Hence, the time-dependent Schrödinger equation is solved by expanding the electronic wave function in a truncated basis of states $\left\{\varphi_{i}\right\}$. A set of first-order coupled differential ordinary equations for the coefficients $\left\{a_{i}\right\}$ originating from this expansion is integrated numerically along the classical trajectory of the projectile for a given impact parameter $b$. The independent particle model is adopted for one active electron moving in the electrostatic field due to the nuclei and other electrons. The other (passive) target electrons are include in a frozen-core Hartree-Fock framework. The partial cross sections $\sigma_{i}$ are obtained by integrating the one-electron probabilities $\left|a_{i}\right|^{2}$ (times the number of target electrons in the corresponding shell) over the impact parameter $b$. Finally, the electronic stopping cross section $S_{e}$ can be computed as

$$
S_{e}=\sum_{i} \sigma_{i} \Delta E_{i}
$$

since each excited, continuum, or projectile-centered state corresponds to a well-defined energy transfer $\Delta E_{i}=E_{i}-E_{0}\left(E_{0}\right.$ is the energy of the initial state).

In order to evaluate the electronic stopping power of hydrogen beams in $\mathrm{He}$ gas, we have to calculate the energy dissipation in three reaction classes:

(i) $\mathrm{H}^{+}+\mathrm{He}^{0} \rightarrow \mathrm{H}^{+}+\mathrm{He}^{*}$ or $\rightarrow \mathrm{H}^{0}+\mathrm{He}^{+}$,

(ii) $\mathrm{H}^{0}+\mathrm{He}^{0} \rightarrow \mathrm{H}^{0}+\mathrm{He}^{*}$,

(iii) $\mathrm{He}^{0}+\mathrm{H}^{0} \rightarrow \mathrm{He}^{0}+\mathrm{H}^{*}$,

where * includes excitation and ionization as well. For case (i), we have evaluated the electronic energy loss due to the electron-capture process. Ionization and excitation of the target electrons have been computed for cases (i) and (ii). Case (iii) provides the energy dissipation by 
projectile electron loss and projectile excitation.

The active-electron-projectile interaction is the Coulomb potential for incoming protons and a hydrogenlike screened potential for $\mathrm{H}^{0}$ and $\mathrm{He}^{0}$ as projectile, with

$$
\begin{array}{r}
V_{p e}(\mathbf{R}-\mathbf{r})=-\left[\frac{Z_{p}-n_{p}}{|\mathbf{R}-\mathbf{r}|}+n_{p}\left[Z_{\mathrm{eff}}+\frac{1}{|\mathbf{R}-\mathbf{r}|}\right]\right. \\
\left.\times \exp \left(-2 Z_{\text {eff }}|\mathbf{R}-\mathbf{r}|\right)\right],
\end{array}
$$

where $Z_{p}$ is the projectile nuclear charge, $n_{p}$ is the number of projectile electrons, and $Z_{\text {eff }}$ is the effective projectile charge as seen by the electrons that are attached to the incident particle. In the present case, we have used $Z_{\text {eff }}=1.69$ for the inverted collision system (He as projectile).

The basis set used in this work consists of targetcentered atomic orbitals. Furthermore, in the case of a bare projectile, a hydrogenlike $1 s$ projectile-centered state is also included:

$$
\varphi_{p}(\mathbf{r}-\mathbf{R}, t)=\frac{2}{\sqrt{4 \pi}} Z_{p}^{3 / 2} e^{-Z_{p}|\mathbf{r}-\mathbf{R}|} e^{i \mathbf{v} \cdot \mathbf{r}} e^{-i \varepsilon_{p} t},
$$

where $\mathbf{R}$ and $\mathbf{v}$ are the relative coordinate and velocity between projectile and target nucleus, respectively. $\varepsilon_{p}$ is the sum of the internal energy of the projectile-centered state and the translational energy $0.5 v^{2}$. This extra state strongly improves the stopping-power calculation at low energies since capture into the projectile-excited states is of minor importance. It should be pointed out that the above projectile-centered state is preorthogonalized in the present calculation by subtracting its projection on all target-centered states [15].

The matrix elements between target-centered and projectile-centered states are calculated by replacing the translation factor $\exp (i \mathbf{v} \cdot \mathbf{r})$ by its Taylor expansion up to the second order around $\mathbf{R}$. This is well justified at low velocities since $\mathbf{v} \cdot \mathbf{r}$ varies sufficiently slowly in the region where $\varphi_{p}$ is most important. This low-energy approximation is very useful, because the use of exact velocitydependent matrix elements would be very time consuming and the electron-capture contributions are only important for low to intermediate velocities. It is emphasized that a Taylor expansion around the mean value of $\mathbf{r}$, as it was used in previous works [15], leads to problems for transitions between the projectile state and the target continuum.

In contrast to other coupled-channel calculations $[11,12]$, we have used a large number of continuum wave packets (up to 224 gerade [11] states with partial waves up to $l=6$ ), since the computation of the stopping power demands high accuracy of the emitted-electron energy spectrum. In addition, the energy loss due to capture into projectile-excited states is naturally included in a large-basis-set calculation. Further details of the calculation may be found in Ref. [11].

\section{RESULTS}

Figure 1(a) shows our coupled-channel atomic-orbital (AO) results for the electronic stopping cross sections

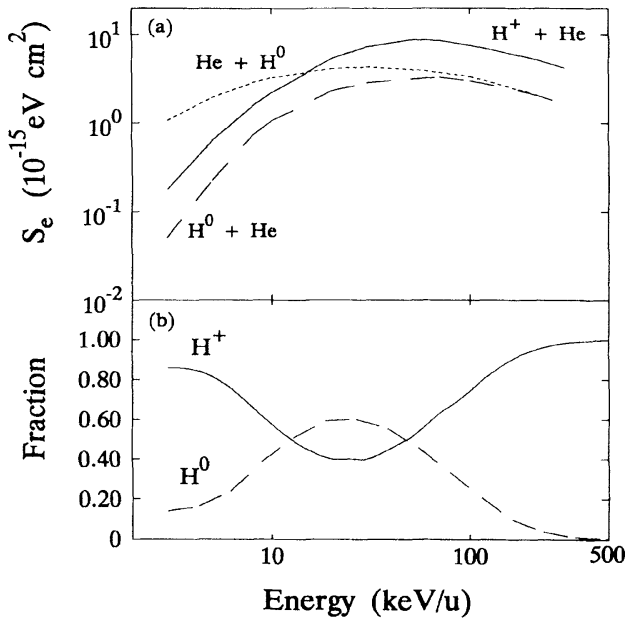

FIG. 1. (a) Coupled-channel results for electronic stopping cross section for $\mathrm{H}^{+}$and $\mathrm{H}^{0}$ beams incident on atomic $\mathrm{He}$ vs incident energy (solid and long-dashed lines). Ionization and excitation of the projectile, in the case of the $\mathbf{H}^{0}$ charge-state fraction, is accounted for by considering the collision system $\mathrm{He}+\mathrm{H}^{0}$ (short-dashed line). (b) Experimental equilibrium fractions for hydrogen beams in helium gas, from Ref. [21].

corresponding to the reaction classes described previously. The energy loss involving neutral collision-partners $\left(\mathrm{H}^{0}+\mathrm{He}\right.$ and $\left.\mathrm{He}+\mathrm{H}^{0}\right)$ is basically due to target or projectile ionization. Excitation of the target or the projectile is of minor importance. The same holds true for collisions between $\mathrm{H}^{+}$and $\mathrm{He}$ at high energies $(E>100$ $\mathrm{keV})$. However, the main contribution for low energies comes from the capture of target electrons into the projectile $1 s$ state. From this figure, we can see that the partial electronic stopping power for bare hydrogen is dominant at high energies, whereas excitation and ionization of the projectile yield the highest partial cross section at low velocities. Nevertheless, the projectile ionization leads to an enhancement of the $\mathbf{H}^{+}$charge-state fraction, and consequently the contribution of $\mathrm{H}^{0}$ to the stopping processes is reduced. The experimental equilibrium fractions [21] for hydrogen beams in $\mathrm{He}$ gas are shown in Fig. 1(b). The $\mathrm{H}^{+}$fraction increases for high and low energies as well. The neutral fraction is only significant for intermediate ion velocities. This means that the $\mathrm{H}^{+}+\mathrm{He}$ collisions also dominate the low-energy part of the stopping power. For energies around $30 \mathrm{keV} / \mathrm{u}$, all reaction classes are equally important.

It is pointed out that we could not estimate the equilibrium fractions of $\mathrm{H}^{+}$and $\mathrm{H}^{0}$ from the present calculation because of the above-described approximation (Taylor expansion) that is involved in the computation of the capture matrix elements. In fact, the low-energy Taylor expansion works quite well only for energies lower than 20 $\mathrm{keV} / \mathrm{u}$. This is not a problem since, for high energies, it is possible to evaluate the total energy loss due to capture and ionization employing a huge basis set [11].

In Fig. 2, the equilibrium mean total stopping cross section per atom for $\mathrm{H}+\mathrm{He}$ collisions is presented in comparison with experimental data of different groups [16-20]. The solid curve represents the values of Fig. 


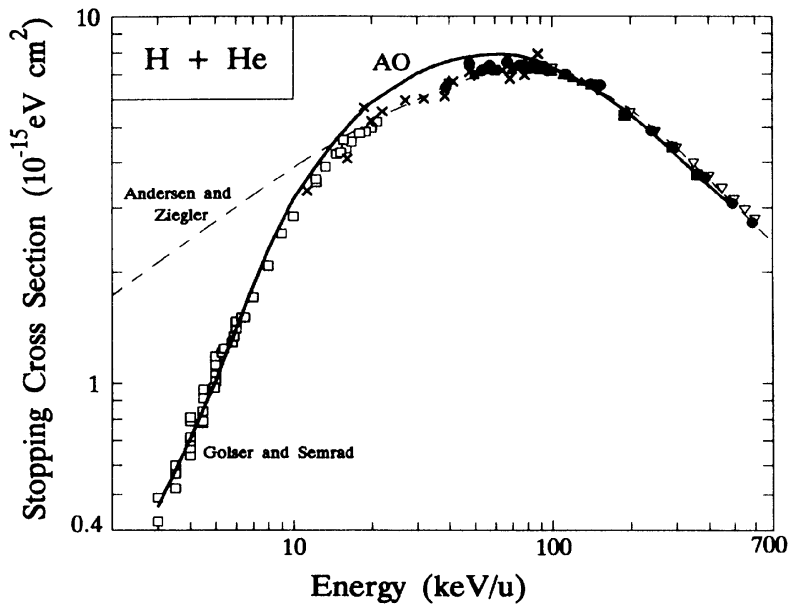

FIG. 2. Equilibrium mean stopping cross section per atom for hydrogen beams penetrating $\mathrm{He}$ gas. Theoretical results: present atomic-orbital (AO) calculation (solid line), Andersen and Ziegler [3] (dashed line). Experimental values: open squares [1], open triangle [16], crosses [17], closed squares [18], closed triangles [19], closed circles [20].

1(a) weighted with the corresponding charge-state fractions from Fig. 1(b) (the contribution due to $\mathrm{H}^{-}$can be neglected [21]). For comparison reasons, we have added the Ziegler-Biersack-Littmark nuclear stopping power [5] for incident deuteron (at low velocities the experiments were carried out with deuterons) to our calculated values. Nevertheless, according to Ref. [5], the nuclear stopping contribution is much less than $3 \%$ for energies higher than $8 \mathrm{keV}$, but can reach $30 \%$ at $3 \mathrm{keV}$.

Special attention should be drawn to the low-energy stopping-power data that was recently measured by Golser and Semrad [1]. At energies below $10 \mathrm{keV}$, experimental and theoretical result agree within $5 \%$ or better. Figure 2 also displays the electronic stopping power as predicted by the semiempirical formula of Andersen and Ziegler [3], which uses velocity proportionality to extrapolate their fit to low energies. The deviation from the velocity proportionality is assigned in Ref. [1] to a threshold effect, i.e., the existence of a finite minimum energy transfer in $\mathbf{H}+\mathrm{He}$ collisions. It was speculated in that work that the slowing down at low velocities is dominated by ionization processes. According to our calculations, the low-energy part of the stopping power is governed by electron capture, which has a minimum energy transfer $\Delta E_{\min }$ of about $\left(0.4+0.5 v^{2}\right)$ a.u. Again, the deviation from a velocity proportionality can be attributed to an influence of a threshold, but for the electroncapture mechanism.

A useful criterion may be given to determine the classical threshold projectile energy $E_{t}$ below which the effect of a minimum energy transfer $\Delta E_{\min }$ becomes important.
If we suppose that the maximum energy transfer between projectile and electron in a binary collision should exceed $\Delta E_{\min }$, we have $E_{t}=M_{p} \Delta E_{\min } / 4\left(M_{p}\right.$ is the projectile mass in units of electron mass). Using this criterion, we estimate a classical threshold energy of $8 \mathrm{keV}$, which is consistent with Fig. 2.

Furthermore, for the investigated case of $Z_{p}<Z_{T}$ and $I_{p}<I_{T}\left(I_{p}\right.$ and $I_{T}$ are the projectile and target ionization potentials, respectively), the electronic stopping cross section $S_{e}$ may be approximated by $S_{e} \approx \sigma_{\text {capture }}\left(I_{T}+v^{2}\right)$ at low velocities. The energy transfer $\left(I_{T}+v^{2}\right)$ accounts for a single capture-and-loss cycle of the dominant $q=1$ charge-state fraction. For homonuclear systems $\left(Z_{p}=Z_{T}\right)$ at low energies, one may write

$$
S_{e} \approx \sigma_{\text {loss }}\left(2 I_{T}+1.5 v^{2}\right)+2 \sigma_{\text {excitation }} \Delta E_{\text {excitation }} .
$$

The corresponding cross sections $\sigma_{\text {loss }}$ and $\sigma_{\text {excitation }}$ for neutral projectiles, and $\sigma_{\text {capture }}$ for singly-charged ions, may be obtained from tabulated atomic cross sections.

At $30 \mathrm{keV} / \mathrm{u}$, we find the largest deviation between the measured stopping power and our calculated values, of about $12 \%$. This may be attributed to an overestimation of cross sections for multielectron processes because of the use of the independent particle model [22]. We emphasize that the present calculation does not properly take into account events in which more than one electron is actively involved, e.g., double target ionization or excitation and simultaneous projectile and target ionization.

\section{CONCLUSIONS}

In summary, a full calculation of the electronic stopping power is performed by considering, in detail, each basic mechanism of energy loss of bare and neutral hydrogen atoms penetrating $\mathrm{He}$ gas. A deviation from the velocity proportionality of the electronic stopping power at low bombarding energies was found in the present work to reproduce the experimental data of Golser and Semrad [1] to within about 5\%. A good overall agreement with experimental results of different groups was also obtained at higher incident energies $(>70 \mathrm{keV} / \mathrm{u}$ ). The remaining discrepancies at energies around $30 \mathrm{keV}$ are assigned to multielectron processes. It is emphasized that these effects will be less important for heavier projectiles and targets, since the electron-electron interactions will be reduced in comparison with the nucleus-electron interactions.

\section{ACKNOWLEDGMENTS}

We would like to thank J. P. Biersack for helpful discussions and a critical reading of the present manuscript. One of the authors (P.L.G.) acknowledges the support given by Conselho Nacional de Desenvolvimento Científico e Tecnológico (CNPq Brazil). 
${ }^{*}$ Permanent address: Universidade Federal do Rio Grande do Sul, Brazil.

[1] R. Golser and D. Semrad, Phys. Rev. Lett. 66, 1831 (1991) ( $d$ and $p$ projectiles were used).

[2] L. C. Northcliffe and R. F. Schilling, Nucl. Data Tables 7, 233 (1970).

[3] The Stopping and Ranges of Ions in Matter, edited by $\mathrm{H}$. Andersen and J. F. Ziegler (Pergamon, New York, 1977), Vol. 3.

[4] F. Janni, At. Data Nucl. Tables 27, 147 (1982).

[5] J. F. Ziegler, J. P. Biersack, and U. Littmark, The Stopping and Ranges of Ions in Solids (Pergamon, Oxford, 1985), Vol. 1.

[6] O. B. Firsov, Zh. Eksp. Teor. Fiz. 36, 1517 (1959) [Sov. Phys. JETP 9, 1076 (1959)].

[7] J. Lindhard, Mat. Fys. Medd. Dan. Vidensk. Selsk. 28, No. 8 (1954); J. Lindhard and M. Scharff, Phys. Rev. 124, 128 (1961); J. Lindhard, M. Scharff, and H. E. Schiott, Mat. Fys. Medd. Dan. Vidensk. Selsk. 33, No. 8 (1963).

[8] P. L. Grande and G. Schiwietz, Phys. Lett. A 163, 439 (1992).

[9] P. M. Echenique, R. M. Nieminen, and R. H. Ritchie, Solid State Commun. 37, 779 (1981); P. M. Echenique, R. M. Nieminen, J. C. Ashley, and R. H. Ritchie, Phys. Rev. A 33, 897 (1986).

[10] P. Sigmund, in Interaction of Charged Particles with Solid and Surfaces, Vol. 271 of NATO Advanced Study Institute,
Series B: Physics, edited by A. Grass-Marti, H. M. Urbassek, N. R. Arista, and F. Flores (Plenum, New York, 1991), and related papers.

[11] G. Schiwietz, Phys. Rev. A 42, 296 (1990); P. L. Grande and G. Schiwietz, ibid. 44, 2984 (1991); G. Schiwietz and P. L. Grande, Nucl. Instrum. Methods B 69, 10 (1992).

[12] J. F. Reading, A. L. Ford, G. L. Swafford, and A. Fitchard, Phys. Rev. A 20, 130 (1979).

[13] L. Willets and S. J. Wallace, Phys. Rev. 169, 84 (1968); M. R. Flannery and K. J. MacCann, Phys. Rev. A 8, 2915 (1973).

[14] U. Wille and R. Hippler, Phys. Rep. 132, 129 (1986).

[15] O. G. Larsen and K. Taulberg, J. Phys. B 17, 4523 (1984).

[16] H. K. Reynolds, D. N. F. Dunbar, W. A. Wenzel, and W. Whaling, Phys. Rev. 92, 742 (1953).

[17] J. A. Phillips, Phys. Rev. 90, 532 (1953) ( $d$ and $t$ projectiles were used for incident energies below $40 \mathrm{keV})$.

[18] P. K. Weyl, Phys. Rev. 91, 289 (1953).

[19] J. T. Park and E. J. Zimmermann, Phys. Rev. 131, 1611 (1963).

[20] F. Besenbacher, H. H. Andersen, P. Hvelplund, and H. Knudsen, Mat. Fys. Medd. Dan Vidensk. Selsk. 40, No. 3 (1979).

[21] S. K. Allison, Rev. Mod. Phys. 30, 1137 (1958).

[22] B. Skogvall and G. Schiwietz, Phys. Rev. Lett. 65, 3265 (1990); Phys. Rev. A 46, 5687 (1992). 